\title{
Minute Splenic Pseudoaneurysm Causing Hemorrhage within a Pancreatic Pseudocyst: The Utility of CT during Splenic Arteriography
}

\author{
Yuko Ogawa ${ }^{1}$, Tsuyoshi Tajima ${ }^{1}$, Yoshitaka Shida ${ }^{1}$, Yasushi Kojima ${ }^{2}$, Tomoyuki Noguchi ${ }^{1}$, Takashi Okafuji ${ }^{1}$, \\ Kanako Murakami ${ }^{1}$, Masatoshi Hotta ${ }^{3}$, Noriaki Wada ${ }^{1}$, Tomotaka Iraha ${ }^{1}$, Kota Yokoyama ${ }^{1}$, Daichi Momosaka ${ }^{1}$ \\ Departments of ${ }^{1}$ Diagnostic Radiology, ${ }^{2}$ Gastroenterology and Hepatology and ${ }^{3}$ Nuclear Medicine, National Center for Global \\ Health and Medicine, Tokyo, Japan.
}

\section{Corresponding Author:}

Dr. Tsuyoshi Tajima

Email: ttajima@hosp.ncgm.go.jp

This is an Open Access article distributed under the terms of the Creative Commons Attribution License (creativecommons.org) licenses/by/3.0).

Received : October 25, 2016

Accepted : February 4, 2017

Published : March 20,2017

\begin{abstract}
Background: Chronic pancreatitis with pseudocyst complicated by hemorrhage has a poor prognosis. Case Report: A 50-year-old Japanese male, known case of pancreatic pseudocyst presented with upper abdominal pain. Emergency CT demonstrated a hyper-attenuated area within the pancreatic pseudocyst, indicating a fresh hemorrhage. However, dynamic contrast-enhanced CT showed neither contrast extravasation nor pseudoaneurysm. Because laboratory data showed an increased serum level of pancreatic enzyme, diagnosis of acute exacerbation of chronic pancreatitis was considered. An arterial hemorrhage within the pancreatic pseudocyst was suspected, and angiography was performed to identify the bleeding site. The splenic angiography initially showed no definite findings of pseudoaneurysm or extravasation. CT during splenic arteriography revealed a splenic artery aneurysm and irregularity of the splenic artery branch at the proximal site of the pseudoaneurysm. Transcatheter arterial embolization was performed to prevent arterial bleeding from the pseudoaneurysm, and the injured segment of the splenic artery was embolized using 12 microcoils with an isolation method. Conclusion: Selective CT arteriography using interventional CT equipment might be useful in the three-dimensional evaluation of a minute or ambiguous visceral aneurysm on transvenous contrast-enhanced CT and selective angiograms.
\end{abstract}

Keywords: Abominal Pain, Aneurysm, Angiography, Embolization, Hemorrhage, Pancreatic Pseudocyst.

\section{Introduction}

Enlargement of pseudocyst leads to incorporation of adjacent arteries into its wall, and weakening of the vessel wall leading to pseudoaneurysm formation. Once a false aneurysm is complicated by arterial hemorrhage, without treatment the patient's prognosis is poor. In such a clinical setting, close inspection to identify the site of bleeding and active treatment are required.

We recently treated a patient with a pancreatic pseudocyst associated with chronic pancreatitis that showed an intracystic hemorrhage.
Herein we report this patient's case and we discuss both the localization of microaneurysms in the visceral artery and the utility of interventional CT in similar cases.

\section{Case Report}

The patient was a 50-year-old Japanese male who was first admitted to a nearby hospital with left upper abdominal pain. He was diagnosed as having a pseudocyst in the pancreatic tail, and he had been followed-up at an outpatient department. Six months later, a sudden abdominal pain developed and he was admitted to our hospital. 
Emergency CT was performed, and he was diagnosed as having a pancreatic pseudocyst with an intracystic hemorrhage. His medical history was unremarkable, but his history of alcohol consumption was notable: he had been drinking approximately $70 \mathrm{mg}$ alcohol/day on 3 days/week for a number of years. He had begun to limit his alcohol consumption since last two months before his emergency admission. His smoking history was also remarkable, and his Brinkman index was 580 .

Regarding the patient's clinical course, because his laboratory data at admission showed an increased serum level of amylase (160 U/L, normal range 30-120 U/L), normal white blood cell (WBC) count $(7,450$, normal range 3,500$9,000 / \mu \mathrm{L}$ ) and normal C-reactive protein (CRP: $0.07 \mathrm{mg} / \mathrm{dL}$, normal range $0-0.3 \mathrm{mg} / \mathrm{dL}$ ), he was diagnosed as having an acute exacerbation of chronic pancreatitis. After his hospitalization, fever occurred and abnormal values of laboratory data such as WBC count $(11,420 / \mu \mathrm{L})$, serum amylase (275 U/L), lactate dehydrogenase (LDH: $402 \mathrm{U} / \mathrm{L}$, normal range 119-229 U/L), and increased C-reactive protein (CRP: $21.18 \mathrm{mg} / \mathrm{dL}$ ) were recognized. An intravenous drip infusion of the combination of sulbactam and ampicillin was started, and then his CRP level began to improve. Because of the sudden onset of abdominal pain during the follow-up for his chronic pancreatitis, the hyper-attenuated contents of the pseudocyst, and splenic artery branches neighboring the pseudocyst, we strongly suspected an intracystic arterial hemorrhage with the explosion of the splenic artery pseudoaneurysm. Based on the deficit of image findings suggesting active bleeding, we administered conservative treatment.

The findings of serial CT images were as follows: (i) contrast-enhanced CT showed a 47-mm unilocular cystic lesion in the pancreatic tail, and the CT value indicated water attenuation [Fig.1a]. (ii) A follow-up CT at 3 months after the image shown in figure $1 \mathrm{~A}$ revealed the regression of the pancreas tail cyst to $40 \mathrm{~mm}$ in diameter [Fig.1b]. (iii) An emergency abdominal CT was performed 6 months after the finding illustrated in figure 1A; the unenhanced CT demonstrated an increase in the size of the cyst (maximum diameter, $5.8 \mathrm{~cm}$ ), and the cyst showed a fluid-fluid level (CT values, 9-44 $\mathrm{HU})$ composed of a hyper-attenuating area in the central region and a hypo-attenuating area in the peripheral region, indicating a fresh hemorrhage [Fig.1c]. An arterial-phase image of contrastenhanced CT showed that there was neither contrast extravasation nor a pseudoaneurysm around the pseudocyst [Fig.1d]. There were no findings suggesting active pancreatitis.

Abdominal MRI was performed on the sixth day of illness. The non-contrast-enhanced MR showed that there was no abnormality in the pancreatic parenchyma. An unilocular cystic mass measuring $60 \times 54 \mathrm{~mm}$ was detected in the pancreas
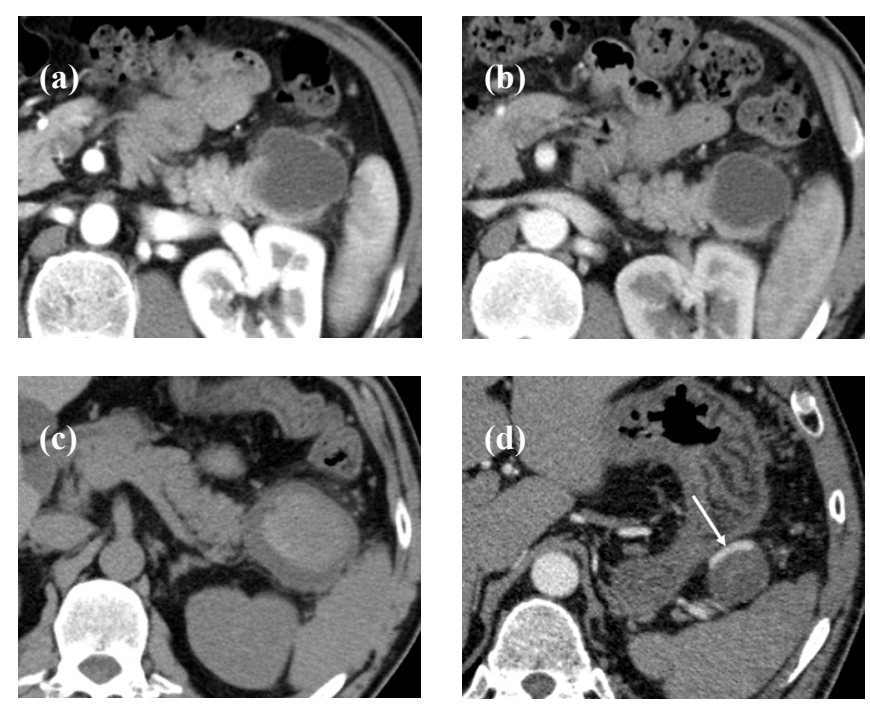

Fig.1: Serial CT images (a): At the onset of the abdominal pain showing 4.7-cm unilocular cystic lesion in the pancreatic tail. (b): 3 months follow up images shows a hypo-attenuating cyst of the pancreas tail which was slightly reduced to 4.0 $\mathrm{cm}$ diameter. $(\mathbf{c}, \mathbf{d}): 6$ months after the image in panel $A$. Unenhanced CT shows an increase in cyst size, and fluidfluid level. Arterial-phase image of contrast-enhanced CT shows no false aneurysm around the pseudocyst. Note the main trunk of the splenic artery is running at the cephalad level of the pancreatic pseudocyst (arrow) (d). 
tail; the mass was hypo- to hyper-intense on T2weighted images and hypo- to hyper-intense in fat-saturated T1-weighted images. Fluid retention developed around the pancreatic tail. The contrast enhancement effect was absent within the mass.

An arterial hemorrhage within the pancreatic pseudocyst was suspected, and we carried out exploratory angiography to identify a bleeding site. Angiography was performed on the $12^{\text {th }}$ day of illness; the selective splenic arteriogram showed no definite findings of pseudoaneurysm or extravasation [Fig.2]. CT during splenic arteriography (CTSA) was then performed. Interventional $\mathrm{CT}$ equipment with 40-detector rows (Siemens Healthineer Japan, Tokyo) was used. For the CTSA, CT scanning was started 7 seconds after the initiation of the infusion of contrast medium: a total of $40 \mathrm{~mL}$ of $50 \%$ diluted $-300 \mathrm{mg} / \mathrm{mL}$ of Oiparomin (Konica Minolta Japan, Tokyo) was used. The CTSA revealed a 4.5-mm-diameter false aneurysm in the branch of the splenic artery [Fig.3a]. In addition, an approximately $2-\mathrm{cm}$ mild irregularity of the splenic artery branch at the proximal site of the pseudo-aneurysm was observed [Fig.3a]. The coronal multiplanar reconstruction (MPR) image of thin-slice contrast-enhanced CT conducted before the angiography [Fig.3b] showed no false aneurysm at the same level as that in figure 3a.

Transcatheter arterial embolization (TAE) was performed to prevent arterial bleeding from the pseudoaneurysm, and both the saccular aneurysm and the injured segment of the splenic artery were embolized using microcoils and an isolation method. After the TAE, the false aneurysm disappeared. The patient's post-TAE course was unremarkable. He recovered and was discharged on the $17^{\text {th }}$ day after admission. Contrast-enhanced CT obtained 5 weeks after the TAE showed a decrease in the CT value within the pseudocyst, and the size of the cyst was slightly decreased. There has been no recurrence of bleeding or symptoms in the subsequent 5-month follow-up.

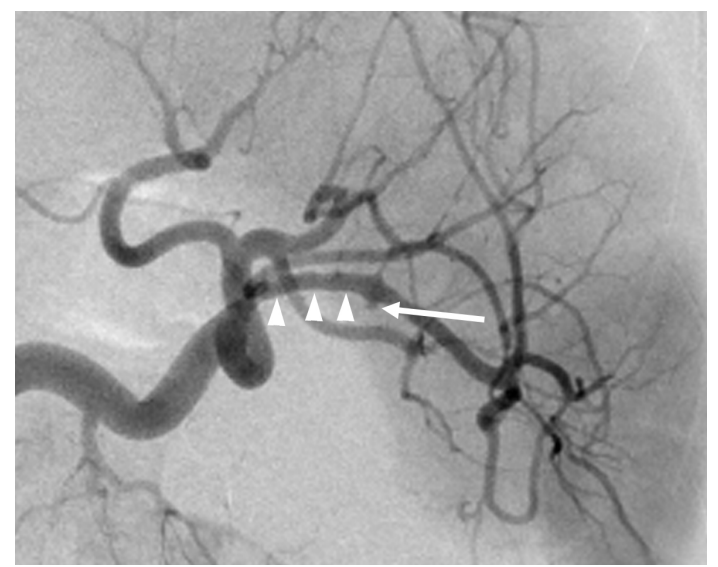

Fig.2: Expanded image of the selective splenic arteriogram. A minute aneurysm in the middle branch of the splenic artery that projects from the splenic artery to the caudal side was detected (arrow), which was judged to be a false aneurysm. A 2-cm mild irregularity of width and narrowness was observed in the splenic artery branch proximal to the pseudoaneurysm (arrowhead).
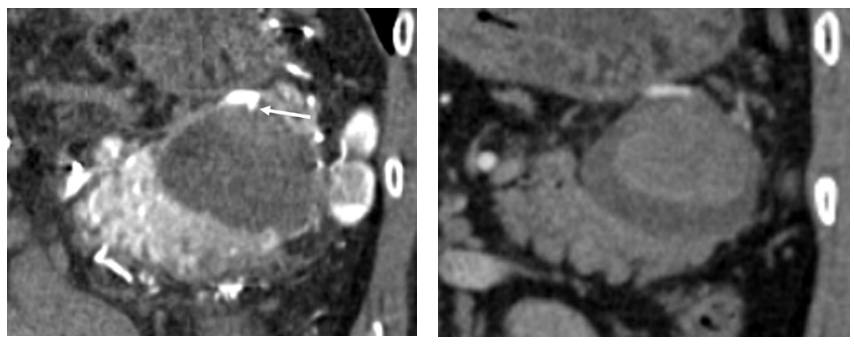

Fig.3: The visualization of the splenic aneurysm was compared between the transvenous CTA and CT during splenic arteriography. (a): The coronal multiplanar reconstruction (MPR) image of $C T$ during selective splenic arteriography shows a false saccular aneurysm in a branch of the splenic artery (arrow). (b): The coronal MPR image of thin-slice contrast-enhanced CT before angiography shows no false aneurysm at the same level as that shown in panel A.

\section{Discussion}

Pancreatic pseudocyst presents as delayed complication ( $\geq 4$ weeks) of pancreatitis [1]. A pancreatic pseudocyst is an encapsulated collection of fluid including amylase from peri-pancreatic tissue and devoid of solid component suggesting pancreatic necrosis [2]. The exact frequency of pancreatic pseudocysts in pancreatitis is unknown, but London [3] reported that CT images obtained 6 
weeks after the onset of acute pancreatitis showed a pseudocyst in $6(5.9 \%)$ of 102 cases. According to a retrospective study of 163 alcoholic individuals with chronic pancreatitis, a treatment intervention was performed for pancreatic pseudocysts in 56 cases (34\%) [3]. Bradley [4] reported that liquid retention was observed in $52(57 \%)$ of 92 cases of acute pancreatitis; 17 (33\%) of these 52 cases became chronic pseudocysts.

Various complications of pancreatic pseudocysts include abscess formation due to the cyst infection, the obstruction or penetration of the neighboring organs, cyst explosion, and intracystic bleeding [5]. A mechanism explaining the development of intracystic bleeding has been considered: a hemorrhage from a false aneurysm created by inflammatory influence or the infiltration of pancreatic enzyme [6-7]. Bleeding pseudocysts were reported to be present in $6 \%-7.5 \%$ of patients with pancreatic pseudocysts [6-7]. Frey [8] noted that intracystic hemorrhage was a complication in $10(7.6 \%)$ of 131 cases of pancreatic pseudocysts, and a false aneurysm was confirmed in $9(90 \%)$ of these 10 cases. According to a review of 98 reported cases of bleeding from a pancreatic pseudocyst and false aneurysm complicated with pancreatitis published over the past 30 years [9], the causal blood vessels were as follows: splenic artery (45\%), gastroduodenal artery $(17 \%)$, inferior pancreaticoduodenal artery (11\%), superior pancreaticoduodenal artery (5\%), superior mesenteric artery $(3 \%)$, hepatic artery $(3 \%)$, left gastric artery $(3 \%)$, right gastric artery (3\%), and dorsal pancreatic artery $(3 \%)$. Less commonly, bleeding from the aorta, the right gastro-epiploic artery, the middle colic artery, the left renal artery, the portal vein and the superior mesenteric vein was also reported. According to Balthazar [10], there have been some surgically explored cases with bleeding pancreatic pseudocysts that showed no false aneurysm or active arterial hemorrhage by angiography; the cause of bleeding was diagnosed as phleborrhagia or diffuse capillary bleeding. Thus, clinicians should pay attention to the bleeding from rare sites such as the veins and portal venous system [9].

When clinical manifestations such as sudden abdominal pain, progression of anemia, hematemesis or melena develop over the course of chronic pancreatitis, a close inspection for an intracystic hemorrhage should be performed. In the reported case, acute arterial bleeding in the pancreatic pseudocyst associated with chronic pancreatitis was suspected because of the intracystic CT value's rise on unenhanced CT and the acute decrease in hemoglobin level and clinical symptoms; however, the contrastenhanced CT simultaneously performed showed no pseudoaneurysm or contrast extravasation.

Generally, if a false aneurysm or active contrast extravasation bleeding is revealed by transvenous contrast-enhanced CT, an interventional procedure using TAE is indicated. Boudfhene [11] performed angiography in hemorrhagic lesions of the pancreatitis and found a false aneurysm in 45 (48\%) of 93 patients. In the same series [11], contrast-enhanced CT detected a false aneurysm in 16 of 73 cases (22\%). Marshall [12] discussed the detection of false aneurysms in five cases with bleeding pancreatic pseudocysts as follows: two cases (40\%) in dynamic CT and four cases $(80 \%)$ in angiography. Kin-Chun [13] performed CT for a close inspection of bleeding from false aneurysms complicated with chronic pancreatitis, and he reported false aneurysms in only four of seven patients (57\%). Frey [6] reported that false aneurysms were confirmed in nine of ten $(90 \%)$ cases of bleeding pancreatic pseudocysts. Thus, the advances in CT technology (including contrast-enhanced CT) and the use of dynamic imaging studies have improved the rate of the detection of false aneurysms in cases of bleeding pancreatic pseudocysts. However, in the present case, the false aneurysm was difficult to identify because of its size, location, overlap with the surrounding structures and the extent of aneurysmal thrombosis. 
The detection accuracy for a tiny false aneurysm may be improved by adding angiographic CT even when the aneurysm is difficult to identify by angiography. Particularly, as in the present case, angiographic CT can be highly useful in the evaluation of a very small aneurysm or an aneurysm at a complicated site such as splenic hilum.

\section{Conclusion}

Exploratory angiography should be immediately performed when an arterial hemorrhage within a pancreatic pseudocyst associated with chronic pancreatitis is strongly suspected, even when the bleeding site is not clear in the transvenous contrast-enhanced CT.

Contributors: YO: Report conception and initial manuscript drafting; TT, YK, TN, TO, KM, MH, NW, TI, KY, DM: Critical review of manuscript; TT: Manuscript revision. All authors were involved in case management. TT will act as guarantor. All authors approved the final version of the manuscript.

Funding: None; Competing interests: None stated.

\section{References}

1. Banks PA, Bollen TL, Dervenis C, Gooszen HG, Colin D. Classification of acute pancreatitis 2012: revision of the Atlanta classification and definition by international consensus. Gut. 2013;62:102-111.

2. Ammann RW, Akovbiantz A, Largiader F, Schueler G. Course and outcome of chronic pancreatitis. Longitudinal study of a mixed medical-surgical series of 245 patients. Gastroenterology. 1984;86:820-828.
3. London NJ, Neoptolemos JP, Lavelle J, Bailey I, James D. Serial computed tomography scanning in acute pancreatitis: a prospective study. Gut. 1989;30:397-403.

4. Bradley EL, Gonzalez AC, Clements JL Jr. Acute pancreatic pseudocysts: incidence and implications. Ann Surg. 1976;184:734-737.

5. Inui K, Irisawa A, Ohara H, et al. Japan Pancreas Society. Guideline for endoscopic treatment of pancreatic pseudocyst 2009. Suizo. 2009;24:571-593. (in Japanese)

6. Bresler L, Boisel P, Grosdidier J. Major hemorrhage from pseudocysts and pseudo aneurysms caused by chronic pancreatitis: surgical therapy. World J Surg. 1991;15:649-653.

7. Sankaran S, Walt S. The natural and unnatural history of pancreatic pseudocyst. Br J Surg. 1975;62:37-44.

8. Frey CF. Pancreatic pseudocyst: operative strategy. Ann Surg. 1978;188:652-662.

9. Stabile BE, Wilson SE, Debas HT. Reduced mortality from bleeding pseudocysts and pseudoaneurysms caused by pancreatitis. Arch Surg. 1983;118:45-51.

10. Balthazar EJ, Fisher LA. Hemorrhagic complications of pancreatitis: radiologic evaluation with emphasis on CT imaging. Pancreatology. 2001;1:306-313.

11. Boudghène $F$, L'Herminé $\mathrm{C}$, Bigot JM. Arterial complications of pancreatitis: diagnostic and therapeutic aspects in 104 cases. J Vasc Interv Radiol. 1993;4:551558.

12. Marshall GT, Howell DA, Hansen BL, Amberson SM, Abourjaily GS, Bredenberg CE. Multidisciplinary approach to pseudoaneurysms complicating pancreatic pseudocysts. Impact of pretreatment diagnosis. Arch Surg. 1996;131:278-283.

13. Chiang KC, Chen TH, Hsu JT. Management of chronic pancreatitis complicated with a bleeding pseudoaneurysm. World J Gastroenterol. 2014;20:1613216137. 